\title{
La construcción de la memoria
}

autobiográfica en niños con TEA mediad por los Sistemas Alternativos y Aumentativos de Comunicación: Una revisión sistemática

\section{Resumen:}

El presente estudio de revisión sistemática se propone analizar el desarrollo de la memoria autobiográfica mediada por el uso de los Sistemas Aumentativos y Alternativos de Comunicación en niños con autismo con deterioro intelectual $y / o$ con deterioro dellenguaje. Para esta revisión se analizaron 22 artículos. Los estudios evidenciaron que en sujetos con TEA a pesar de poder acceder a recuerdos autobiográficos, los mismos pueden presentarse escasos, con dificultad en el autorreferenciamiento, desorganizados o con detalles sensoriales no relevantes. Los análogos visuales a nivel comunicacional son una ayuda relevante para facilitar el acceso a los recuerdos y su organización.

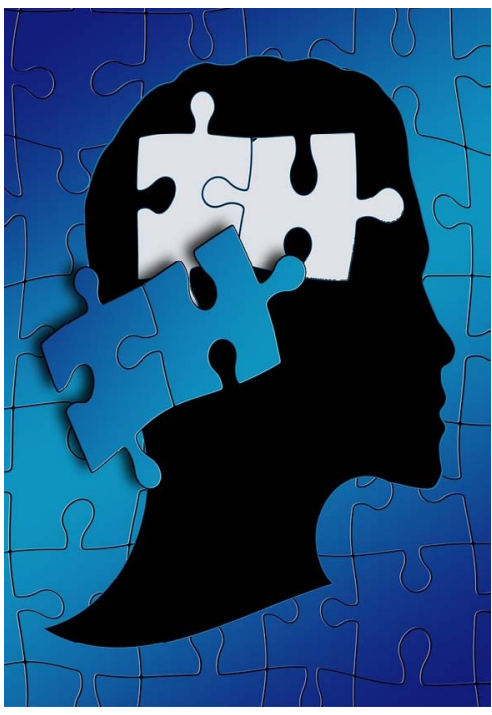

The present systematic review study aims to analyze the development of autobiographical memory mediated by the use of Augmentative and Alternative Communication Systems in children with autism with intellectual impairment and / or language impairment. For this review, 22 articles were analyzed. The studies showed that in subjects with ASD, despite being able to access autobiographical memories, they can be scarce, with difficulty in self-referencing, disorganized or with non-relevant sensory details. Visual analogues at the communicational level are a relevant aid to facilitate access to memories and their organization.

Keywords: Memory, Autism, Communication
Palabras clave: Memoria, Autismo, Comunicación

Dra. María Cecilia Roma mroma5@abc.gob.ar / https://orcid.org/0000-0003-3374-6709 Instituto Superior de Formación Docente No 99 Universidad de Flores.Doctora en Ciencias de la Educación (UBA). Magíster en Psicología Cognitiva y Aprendizaje (FLACSO). Especialista en educación digital inclusiva. Diplomada superior en Necesidades Educativas Especiales, Prácticas Inclusivas y Trastornos del Desarrollo (FLACSO). Docente de nivel superior. Autora de artículos en revistas educativas. Enviado 15/07/20 Publicado 24/10/20 


\section{Introducción}

El presente estudio emerge de interrogantes despertados por la lectura del artículo de Santamaría y Montoya (2010) en el cual se aborda el tema de la memoria autobiográfica y su vínculo entre el yo y el lenguaje. A partir de este estudio surge el interés de indagar el desarrollo de la memoria autobiográfica mediada por el uso de la comunicación alternativa en niños con Trastornos del Espectro Autista (TEA) con deterioro del lenguaje y/o con deterioro intelectual según caracterización del DSM 5 (2014). También se tomó como base para la elaboración de este estudio la tesis doctoral de Solcoff (2011) la cual ofrece un amplio sostén bibliográfico con relación a la memoria de fuente en niños con autismo.

Antes que nada es fundamental diferenciar memoria episódica de memoria autobiográfica. Tulving $(2002,2005)$ destaca la existencia de por lo menos tres elementos constitutivos de la memoria episódica: el sentido subjetivo del tiempo, la conciencia autonoética y el yo, responsable de desplazarse hacia el pasado y hacia el futuro a partir del sentido subjetivo del tiempo de forma autonoética. Esto es, la sensación de reexperimentar el pasado simultáneamente al acto de recuerdo diferente del conocimiento o pensamiento sobre el hecho a recordar, vacío de la experiencia de revivir el evento (Tulving, 1983, 1985, 2002).

Por su parte Katherine Nelson (2003) describe la memoria autobiográfica como un subtipo de memoria episódica. La memoria autobiográfica se constituye como el tipo de memoria que implica un sentido del yo (self) que experimenta un evento ubicado en un determinado punto del espacio y del tiempo, que se encuentra racionado con sucesos significativos para el sujeto producto de emociones, motivaciones e interacción con pares.

De este modo, solamente los eventos relevantes que marcan hitos significativos para el sujeto son los que pasarán a conformar su historia de vida (Nelson, 1993) y son estos mismos eventos los que definen y sitúan al yo, cultural e históricamente (Neisser, 1988).

Diversos autores (Hudson, 1990; Nelson, 1993; entre otros) defienden la idea de que la memoria autobiográfica emerge cuando los niños comienzan a interactuar lingüísticamente sus vivencias personales con otros.

Entre los tres y los seis ańos, el lenguaje le proporciona al sujeto un marco narrativo a través del cual organizar su experiencia, de este modo un determinado suceso pasa a construir su historia personal (por ejemplo Reese y Fivush, 1993). La memoria autobiográfica entonces, hacia aproximadamente los 4 años, se desarrollaría en conjunto con la posibilidad de compartir recuerdos, con e lenguaje como mediador narrativo y con la fuerza de un nuevo yo. En este contexto, el acontecimiento coincidiría con el desarrollo de las actividades mentalistas (Santamaría y Montoya, 2008).

Millward, Powell, Messer y Jordan (2000) sostienen que existe una deficiencia en la constitución de la memoria autobiográfica de las personas con TEA y que ese déficit persiste durante toda la vida. Investigaciones como las de Boucher y Bowler (2008) también demuestran que en la población con TEA se manifiesta un déficit específico en memoria episódica.
Powell y Jordan (1993) todavía sugieren que en los trastornos del espectro autista se problematiza el desarrollo de la memoria de experiencias que impliquen un compromiso directo con el yo (self) (Powell y Jordan, 1993; Hare, Mellor y Azmi, 2007) siendo que es requisito la constitución de este self para el desarrollo de una memoria episódica (Solcoff, 2011).

En el DSMV se explica que el Trastorno de Espectro Autista se caracteriza principalmente por un deterioro en la comunicación einteracción social y patrones de conducta intereses acotados. Las deficiencias lingüísticas pueden abarcar desde la ausencia del habla completamente, ecolalia o lenguaje literal. Su desarrollo también depende del deterioro intelectual. Aunque la capacidad de habla no presente dificultades, su función social recíprocase encontraría afectada (DSMV, 2014).

Como alternativa comunicativa se suelen utilizar instrumentos de intervención par organizar y llevar adelante la comunicación. Estos instrumentos se caracterizan por ser no vocales, se componen de un sistema de signos como gestos naturales, señas, imágenes, fotos entre otros (Tamarit, 1993). Son alternativos al habla y también presentan la particularidad de ser aumentativos dado que pueden favorecer la manifestación de habla (Valdez, 2009).

Valdez explica que el uso de un sistema analógico de comunicación, como son los Sistemas Alternativos y Aumentativos de Comunicación producen cambios en la vida cotidiana del sujeto, especialmente porque permiten ordenar secuencialmente y cronológicamente eventos y por lo tanto planificar a futuro (Valdez, 2009).

Ahora, si el lenguaje es una capacidad quese encuentra comprometida en los trastornos de espectro autista con deterioro del lenguaje y/o intelectual y la comunicación se desenvuelve a partir de análogos como es la comunicación alternativa, se genera el interrogante sobre el papel que juega este tipo de comunicación en el desarrollo de la memoria autobiográfica en la población en cuestión.

\section{Objetivo}

Revisar sistemáticamente estudios significativos que abordan la temática del desarrollo de la memoria autobiográfica mediada por el uso de los Sistemas Aumentativos y Alternativos de Comunicación (SAAC) en niños con Trastornos del Espectro Autista (TEA) con deterioro del lenguaje y/o con deterioro intelectual.

\section{Método}

El presente estudio se valió del uso del método PRISMA, siguiendo los pasos de calidad para la revisión sistemática. Se han utilizado los ítems 1 (título), 2 (resumen), 3 (justificación), 4 (objetivos), 6 (criterio de elegibilidad), 7 (fuentes), 8 (estrategia), 9 (selección y exclusión), 10 (método), 17 (selección), 18 (narración), 23 (resumen de evidencia), 24 (sesgos) y 25 (conclusiones) propuestos por esta metodología. Los ítems que no se tomaron en cuenta han sido el número 5 (protocolo y registro), 11 (lista de variables), 12 (riesgo de sesgo en los estudios individuales), 13 (medidas de resumen), 14 (síntesis de resultados), 15 (riesgos adicionales), 16 (análisis adicionales), 19 (riesgo de sesgo en los estudios) y 22 (riesgo de sesgo entrelos estudios), fueron excluidos dado que refieren a estudios de corte metaanalítico, y difieren de los objetivos de este estudio. Los items vinculados con aspectos financieros (26 y 27) también fueron excluidos dado que se careció de aportes.

Se realizó una búsqueda exhaustiva de literatura publicada acerca del desarrollo de la memoria autobiográfica mediada por 
sistemas de comunicación alternativa (SAAC) en niños con Trastornos del Espectro Autista (TEA) con deterioro del lenguaje y/o con deterioro intelectual.

Para la selección de artículos se han considerado las diferencias terminológicas entre el DSM IV y el DSM V. Durante los años de vigencia del DSM IV, es decir, desde el año 1994 hasta el 2000 y DSM IVTR desde el 2000 hasta el año 2013, se presenta una diferencia en los artículos publicados, el abordaje centrado en autismo o en Asperger. Ante esta diferenciación diagnóstica se considerarán los artículos enfocados en autismo. Desde la vigencia del DSM V, desde el año 2013, se hace referencia a TEA con y sin deterioro intelectual, con y sin deterioro del lenguaje, en este caso se considerarán los artículos que aborden la caracterización de TEA con deterioro del lenguaje y/o con deterioro intelectual.

Las búsquedas bibliográficas se han efectuado en bases de datos con destacado reconocimiento académico. Las mismas ofrecen un vasto material, en inglés, español, francés, ruso y portugués, siendo además de acceso libre y gratuito. Esta búsqueda se realizó en las siguientes bases de datos: REDIB - Red Iberoamericana de Innovación y Conocimiento Científico; DOAJ - Directorio de Revistas de Acceso Abierto; Redalyc -Red de Revistas Científicas de América Latina y el Caribe, España y Portugal; SciELO Scientific Electronic Library Online; Dialnet; Portal Regional en Salud Organización Panamericana de la Salud y British jornal ofpsychology.

\section{Procedimiento}

Para la estrategia de búsqueda se introdujeron los siguientes descriptores en título, abstract o palabras clave: Autobiographical memory + autism; Memoria autobiográfica + autismo; Comunicación + autismo; Communication + autism; Memoria +autismo+comunicación; Communication + autism + memory. Esta combinación de vocabulario específico se pensó en función de ampliar la variedad de artículos vinculados con la temática debido a que la bibliografía específica que investigue los temas en cuestión, es decir, los sistemas alternativos de comunicación para la estimulación de la memoria autobiográfica en sujetos con TEA son escasos. De este modo, la articulación de las palabras clave en las diferentes combinaciones permitió obtener mayor cantidad de artículos relevantes con características de análisis propios de cada investigación que en conjunto permiten obtener una visión general del estado actual de la temática.

La selección de buscadores se debe a la intención de analizar artículos con diferentes enfoques sobre el tema. Para ampliar la variedad de artículos se han seleccionados descriptores en inglés y español, aunque también se han encontrado artículos en idioma portugués, francés y un artículo en ruso.

A continuación se detalla el procedimiento de búsqueda y sus resultados. Se han seleccionado un total de 22 artículos con relación directa con el tema de revisión.

\section{Resultados}

Términos de búsqueda: Autobiographical memory + autism
REDIB Red Iberoamericana de Innovación y Conocimiento Científico

\begin{tabular}{llll}
\hline $\begin{array}{l}\text { DOAJ Directorio de re- } \\
\text { vistas de acceso abierto }\end{array}$ & 7 & $\begin{array}{l}3 \text { se desestiman por } \\
\text { no ser pertinentes }\end{array}$ & $\begin{array}{l}4 \\
\text { seleccionados } \\
\text { incluyendo } 1 \\
\text { artículo en uso }\end{array}$ \\
\hline $\begin{array}{l}\text { Redalyc Red de Revistas } \\
\text { Científicas de América } \\
\text { Latina y el Caribe, España } \\
\text { y Portugal }\end{array}$ & 0 & \\
\hline $\begin{array}{l}\text { SciELO - Scientific Elec- } \\
\text { tronic Library Online }\end{array}$ & 0 & \\
\hline Dialnet & 0 & \\
\hline $\begin{array}{l}\text { Portal Regional en Salud } \\
\text { Organización Panameri- } \\
\text { cana de la Salud }\end{array}$ & 0 & \\
\hline $\begin{array}{l}\text { British Jornal of Psycho- } \\
\text { logy }\end{array}$ & 914 & $2010-2020$ & $2015-220$ \\
& & 328 artículos & \\
\end{tabular}

Términos de búsqueda: Memoria autobiográfica + autismo 
REDIB Red

0

Iberoamericana de

Innovación y

Conocimiento

Científico

DOAJ Directorio

de revistas de

acceso abierto

Redalyc Red de

Revistas

Científicas de

América Latina y

el Caribe, España y

Portugal

SciELO -

Scientific

Electronic Library

Online

Dialnet

Portal Regional en 0

Salud

Organización

Panamericana de la

Salud

British Jornal of

Psychology preseleccionado

1 seleccionado

Término de búsqueda: Comunicación + autismo

\section{REDIB Red}

0

Iberoamericana

de Innovación y

Conocimiento

Científico

$\begin{array}{llll}\text { DOAJ Directorio } & 40 & \text { preseleccionados } & 0 \text { seleccionados } \\ \text { de revistas de } & & 16 & \end{array}$

de revistas de

acceso abierto

Redalyc Red de 1 no pertinente

Revistas

Científicas de

América Latina y

el Caribe, España

y Portugal

SciELO -

59

Scientific

59

9

preseleccionados 1seleccionado

por pertinencia

Revista Perspectivas Metodológicas | Universidad Nacional de Lanús | ISSN 2618-4125
Electronic

Library Online

Dialnet

Portal Regional 9

en Salud

preseleccionados 1 por pertinencia

Organización

Panamericana de

la Salud

British Jornal of 5 no pertinentes

Psychology

Término de búsqueda: Communication + autism

REDIB Red

0

Iberoamericana

de Innovación y

Conocimiento

Científico

\begin{tabular}{llll}
\hline DOAJ Directorio & 1.298 & Idioma: Inglés & 2 son pertinentes \\
de revistas de & & Communication & \\
acceso abierto & & + autism + \\
& memory & \\
& & Preseleccionados \\
& & 52 \\
\hline
\end{tabular}

Redalyc Red de $\quad 0$

Revistas

Científicas de

América Latina y

el Caribe, España

y Portugal

SciELO -

174

preseleccionados 2015-2019

$\begin{array}{lll}\text { Scientific } & 37 & 21 \text { resultados }\end{array}$

Electronic 3 seleccionados

Library Online

Dialnet 0

Portal Regional $34 \quad 0$ pertinentes

en Salud

Organización

Panamericana de

la Salud

British Jornal of 2010-2020 2015-2020 3 seleccionados

Psychology 12.124 resultados 90 resultados

Término de búsqueda: Memoria +autismo + comunicación

Revista Perspectivas Metodológicas | Universidad Nacional de Lanús | ISSN 2618-4125 
REDIB Red

Iberoamericana

1 seleccionado

de Innovación y

Conocimiento

Científico

DOAJ Directorio 2 duplicados

de revistas de

acceso abierto

Redalyc Red de

Revistas

Científicas de

América Latina y

el Caribe, España

y Portugal

SciELO 2

1 duplicado

Scientific

1 no pertinente

Electronic

Library Online

\begin{tabular}{ll} 
Dialnet & 0 \\
\hline Portal Regional & 0
\end{tabular}

Portal Reg

en Salud

Panamericana de

la Salud

British Jornal of 1 no pertinente

Psychology

Término de búsqueda: Communication + autism + memory

REDIB Red

Iberoamericana

de Innovación y

Conocimiento

Científico

\begin{tabular}{llll}
$\begin{array}{l}\text { DOAJ Directorio } \\
\text { de revistas de }\end{array}$ & 52 & $\begin{array}{l}\text { Estudios no } \\
\text { pertinentes a la } \\
\text { temática }\end{array}$ & $\begin{array}{l}2 \text { pertinentes } \\
\text { (seleccionados en } \\
\text { la búsqueda } \\
\text { Communication } \\
+ \text { autism) }\end{array}$ \\
& & & \\
\hline
\end{tabular}

Redalyc Red de

Revistas

Científicas de

América Latina y

el Caribe, España

y Portugal

Revista Perspectivas Metodológicas | Universidad Nacional de Lanús | ISSN 2618-4125

\begin{tabular}{|c|c|c|c|}
\hline $\begin{array}{l}\text { SciELO - } \\
\text { Scientific } \\
\text { Electronic } \\
\text { Library Online }\end{array}$ & 4 & $\begin{array}{l}3 \text { duplicados } \\
1 \text { no pertinente }\end{array}$ & \\
\hline Dialnet & 0 & & \\
\hline $\begin{array}{l}\text { Portal Regional } \\
\text { en Salud } \\
\text { Organización } \\
\text { Panamericana de } \\
\text { la Salud }\end{array}$ & 0 & & \\
\hline $\begin{array}{l}\text { British Jornal of } \\
\text { Psychology }\end{array}$ & $\begin{array}{l}8,576 \text { resultados } \\
2015-2020 \\
2.246 \text { resultados }\end{array}$ & $\begin{array}{l}2018-2020 \\
1086 \text { resultados } \\
2019-2020 \\
692 \text { resultados }\end{array}$ & $\begin{array}{l}\text { Communication } \\
+ \text { autism }+ \\
\text { autobiographical } \\
\text { memory } \\
2015-2020 \\
103 \text { resultados } \\
4 \text { seleccionados }\end{array}$ \\
\hline
\end{tabular}

Diagramas de Flujo

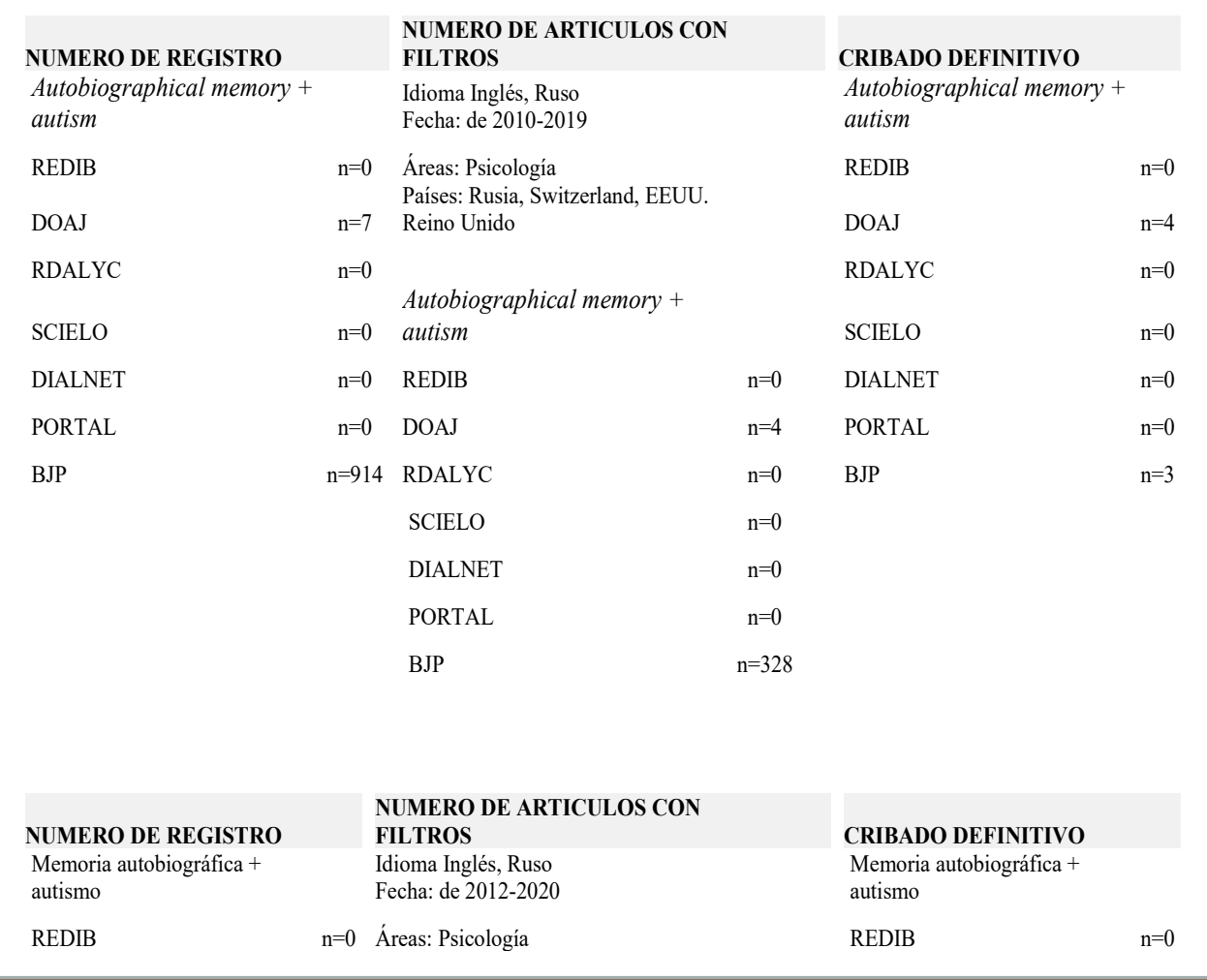

Revista Perspectivas Metodológicas | Universidad Nacional de Lanús | ISSN 2618-4125 


$\begin{array}{lll}\text { DOAJ } & \mathrm{n}=0 & \text { Paises: Reino Unido } \\ \text { RDALYC } & \mathrm{n}=0 & \text { Memoria autobiográica }+ \text { autismo } \\ \text { SCIELO } & \mathrm{n}=0 & \text { REDIB } \\ \text { DIALNET } & \mathrm{n}=0 & \text { DOAJ } \\ \text { PORTAL } & \mathrm{n}=0 & \text { RDALYC } \\ \text { BJP } & \mathrm{n}=1 & \text { SCIELO } \\ & \text { DIALNET } \\ & \text { PORTAL } \\ & \text { BJP }\end{array}$

DOAJ $\quad \mathrm{n}=0$

RDALYC

SCIELO

DIALNET

PORTAL

BJP

NÚMERO DE
REGISTRO

NÚMERO DE ARTICULOS CON FILTROS

CRIBADO

Idioma: Inglés, Portugués, Español.

Comunicación y autism

$\mathrm{n}=0 \quad$ Áreas: Psicología

REDIB

Paises: Reino Unido, Argentina, Chile, Brasil, Italia,

DOAJ

$\mathrm{n}=1 \quad$ Comunicación y autismo

SCIELO

$\mathrm{n}=59 \quad$ REDIB

DIALNET $\quad \mathrm{n}=0$ DOAJ

PORTAL $\mathrm{n}=9$ RDALYC

$\mathrm{n}=5 \quad$ SCIELO

DIALNET

PORTAL

BJP

\begin{tabular}{lll}
\multicolumn{3}{c}{$\begin{array}{l}\text { Comunicación y } \\
\text { autismo }\end{array}$} \\
REDIB & $\mathrm{n}=0$ \\
& DOAJ & $\mathrm{n}=0$ \\
& RDALYC & $\mathrm{n}=0$ \\
$\mathrm{n}=0$ & SCIELO & $\mathrm{n}=1$ \\
$\mathrm{n}=16$ & DIALNET & $\mathrm{n}=0$ \\
$\mathrm{n}=0$ & PORTAL & $\mathrm{n}=1$ \\
$\mathrm{n}=14$ & BJP & $\mathrm{n}=0$
\end{tabular}

NÚMERO DE
REGISTRO

NÚMERO DE ARTICULOS CON

FILTROS

CRIBADO DEFINITIVO

Communication
+autism

Idioma: Inglés, Portugués, Español.

REDIB

$\mathrm{n}=0 \quad$ Áreas: Psicología

DOAJ $\mathrm{n}=1298$ Paiscs: Reino Unido, Argentina, Chile,

REDIB

DOAJ $(C a m-n=0$

autism + memory)

RDALYC $\mathrm{n}=1 \quad$ Communication + autism

RDALYC

SCIELO $\quad \mathrm{n}=174 \quad$ REDIB

DIALNET $\quad \mathrm{n}=34 \quad$ DOAJ (Communication + autism +

PORTAL

$\mathrm{n}=34 \quad$ memory

$\mathrm{n}=9 \quad$ RDALYC

$\mathrm{n}=0 \quad$ SCIELO

BJP

SCIELO
$(2015-2019)$

DIALNET

$\mathrm{n}=52 \quad$ DIALNET

PORTAL

BJP

$\mathrm{n}=0$

Revista Perspectivas Metodológicas | Universidad Nacional de Lanús | ISSN 2618-4125

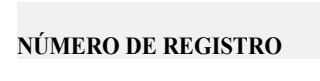

Memoria

REDIB

DOAJ

PORTAL

BJP
$(2015-2020)$

RDALYC

SCIELO

DIALNET

PORTAL

BJP

FILTROS

CRIBADO DEFINITIVO

Idioma: Inglés, Español.

$\mathrm{n}=3$ Áreas: Psicología

$\mathrm{n}=2$ Países: Perú, EEUU

$\mathrm{n}=0$ Memoria +autismo+comunicación

$\mathrm{n}=2$ REDIB

$\mathrm{n}=0$ DOAJ

$\mathrm{n}=0$ RDALYC

$\mathrm{n}=1 \quad$ SCIELO

DIALNET

PORTAL

BJP

Memoria
tautismo

REDIB

DOAJ

RDALYC

$\mathrm{n}=1 \quad$ SCIELO

$\mathrm{n}=0 \quad$ DIALNET

$\mathrm{n}=0 \quad$ PORTAL

$\mathrm{n}=0 \quad$ BJP

$\mathrm{n}=0$

$\mathrm{n}=0$

\begin{tabular}{|c|c|c|c|c|c|}
\hline NÚMERO DE REGISTRC & & $\begin{array}{l}\text { NÚMERO DE ARTICULOS } \\
\text { CON FILTROS }\end{array}$ & & CRIBADO DEFINITIVO & \\
\hline $\begin{array}{l}\text { Communication + } \\
\text { autism + memory }\end{array}$ & & $\begin{array}{l}\text { Idioma: Inglés, Portugués, } \\
\text { Español. } \\
\text { Fecha: de 2010-2020 }\end{array}$ & & Communication + autism & \\
\hline REDIB & $\mathrm{n}=0$ & $\begin{array}{l}\text { Áreas: Psicología } \\
\text { Países: Reino Unido, Argentina, }\end{array}$ & & REDIB & $\mathrm{n}=0$ \\
\hline DOAJ & $\mathrm{n}=52$ & $\begin{array}{l}\text { Chile, Brasil, Italia, EEUUU. } \\
\text { Communication + autism }\end{array}$ & & DOAJ & $\mathrm{n}=2$ \\
\hline RDALYC & $\mathrm{n}=0$ & +memory & & RDALYC & $\mathrm{n}=0$ \\
\hline SCIELO & $\mathrm{n}=4$ & REDIB & $\mathrm{n}=0$ & SCIELO & $\mathrm{n}=0$ \\
\hline DIALNET & $\mathrm{n}=34$ & DOAJ & $\mathrm{n}=2$ & DIALNET & $\mathrm{n}=0$ \\
\hline PORTAL & $\mathrm{n}=9$ & $\begin{array}{l}\text { RDALYC } \\
\text { SCIELO }\end{array}$ & $\begin{array}{l}\mathrm{n}=0 \\
\mathrm{n}=0\end{array}$ & PORTAL & $\mathrm{n}=0$ \\
\hline \multirow[t]{3}{*}{$\begin{array}{l}\text { BJP } \\
(2015-2020)\end{array}$} & $\begin{array}{l}\mathrm{n}=8.576 \\
\mathrm{n}=2.246\end{array}$ & DIALNET & $\mathrm{n}=0$ & $\begin{array}{l}\text { BJP } \\
\text { Communication }+ \text { autism }+\end{array}$ & $\mathrm{n}=103$ \\
\hline & & PORTAL & $\mathrm{n}=0$ & $\begin{array}{l}\text { autobiographical memory } \\
(2015-2020)\end{array}$ & $\mathrm{n}=4$ \\
\hline & & $\begin{array}{l}\mathrm{BIP}(2018-2020) \\
(2019-2020)\end{array}$ & $\begin{array}{r}\mathrm{n}=1.086 \\
\mathrm{n}=692\end{array}$ & Seleccionados & \\
\hline
\end{tabular}

Revista Perspectivas Metodológicas | Universidad Nacional de Lanús | ISSN 2618-4125 


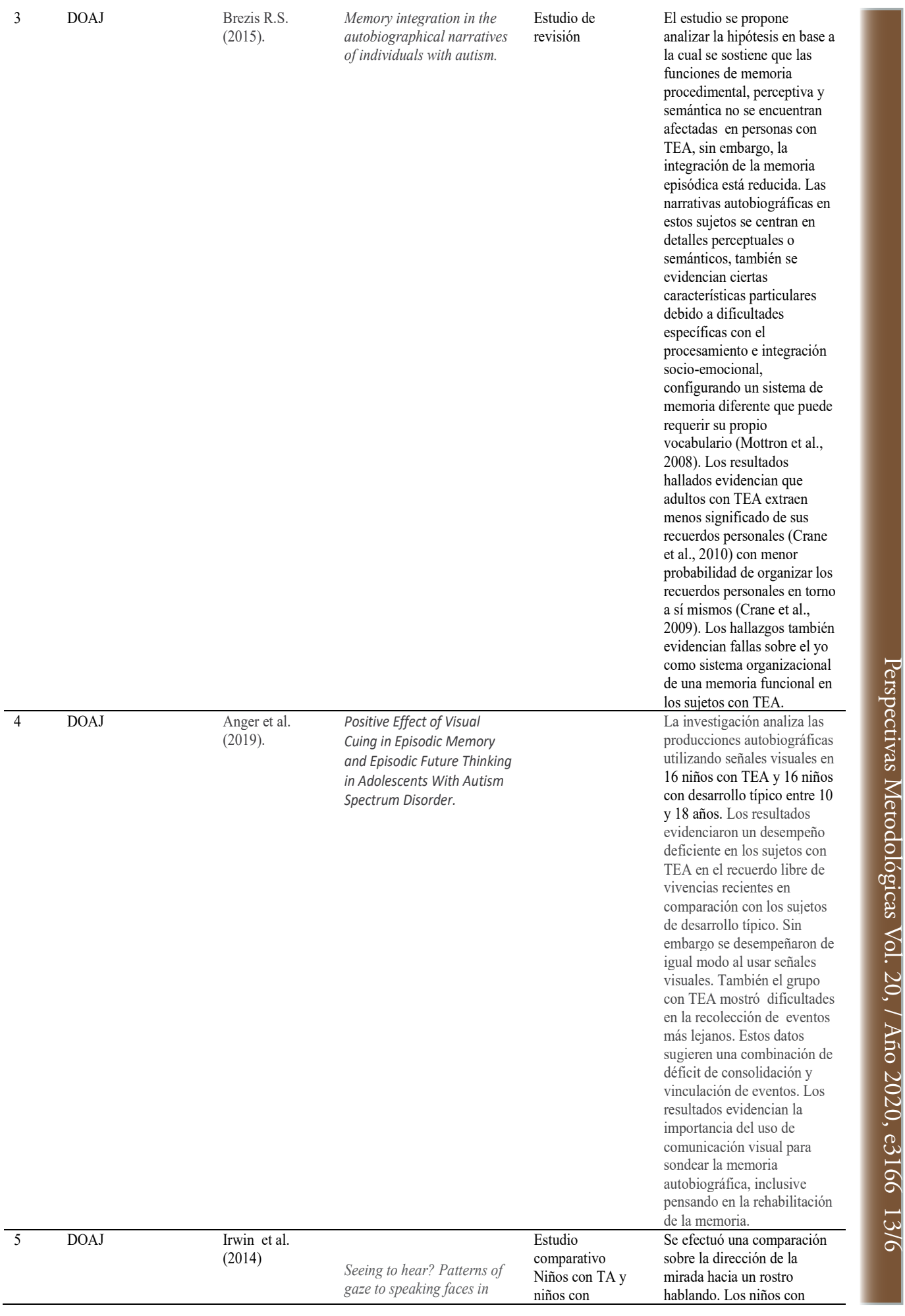

\section{Discusión}

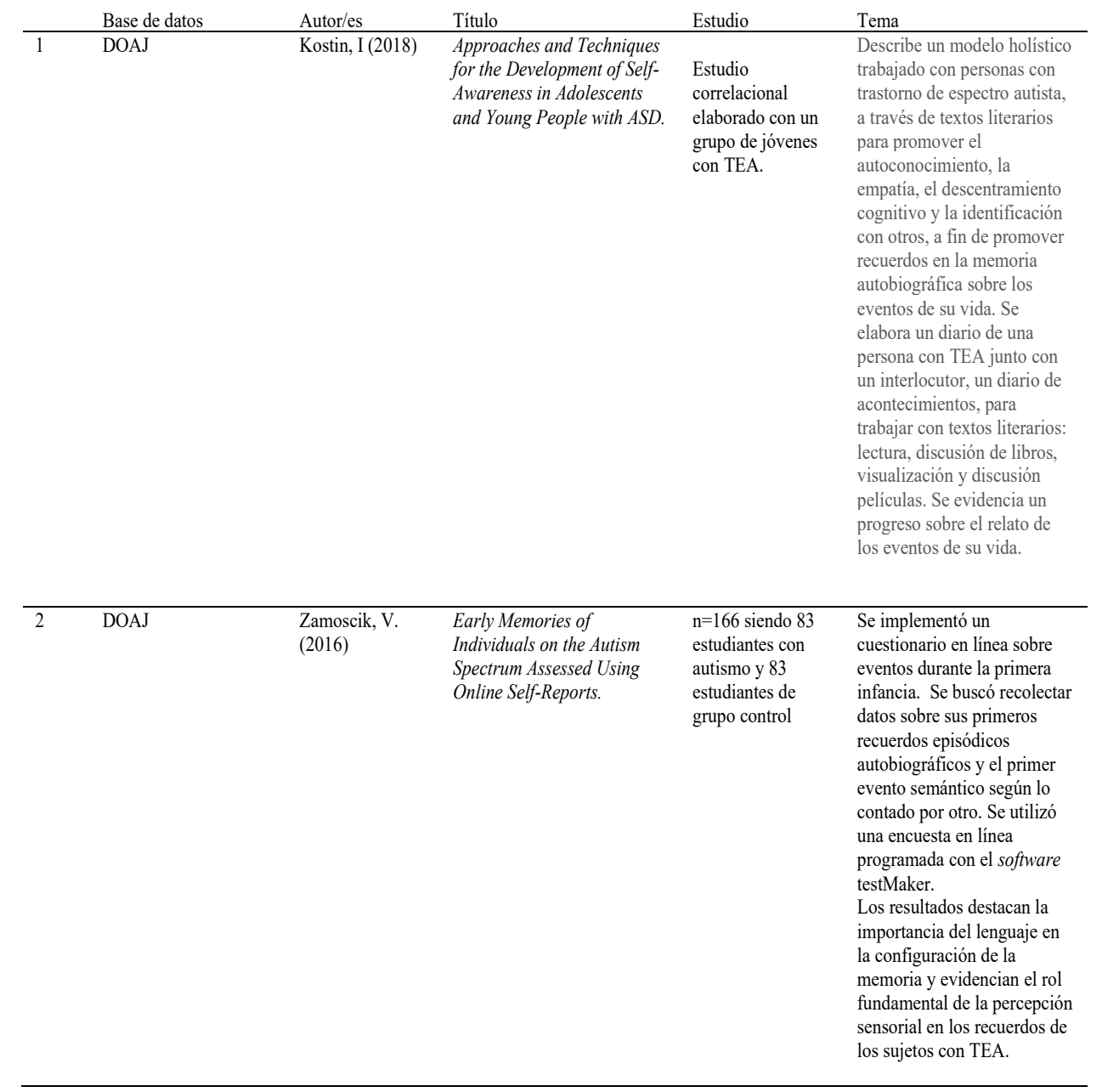

Revista Perspectivas Metodológicas | Universidad Nacional de Lanús | ISSN 2618-4125

23.337

TOTAL DE ARTÍ́CULOS RELEVADOS

22 

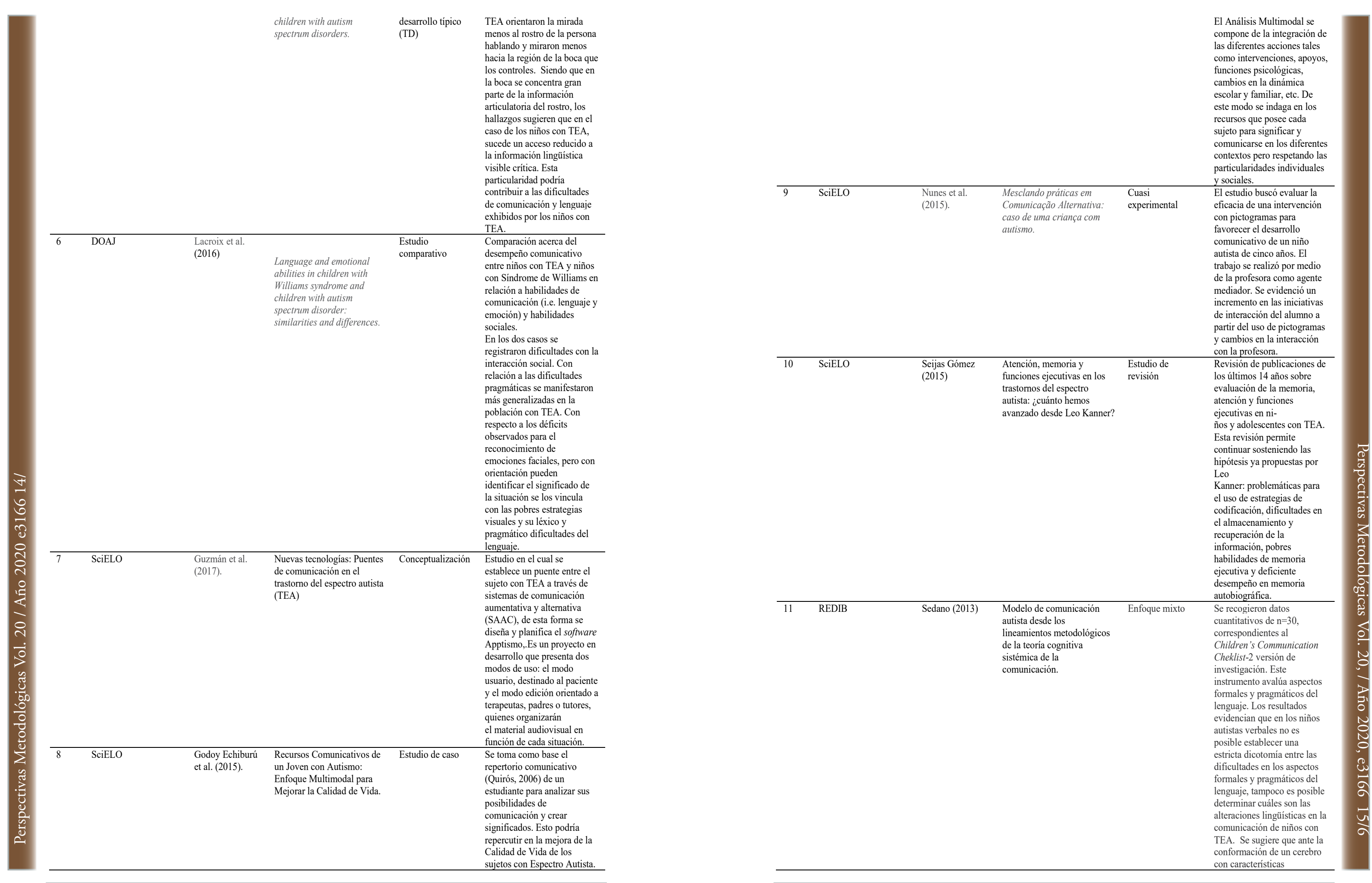

Revista Perspectivas Metodológicas | Universidad Nacional de Lanús | ISSN 2618-4125|

Revista Perspectivas Metodológicas | Universidad Nacional de Lanús | ISSN 2618-4125| 

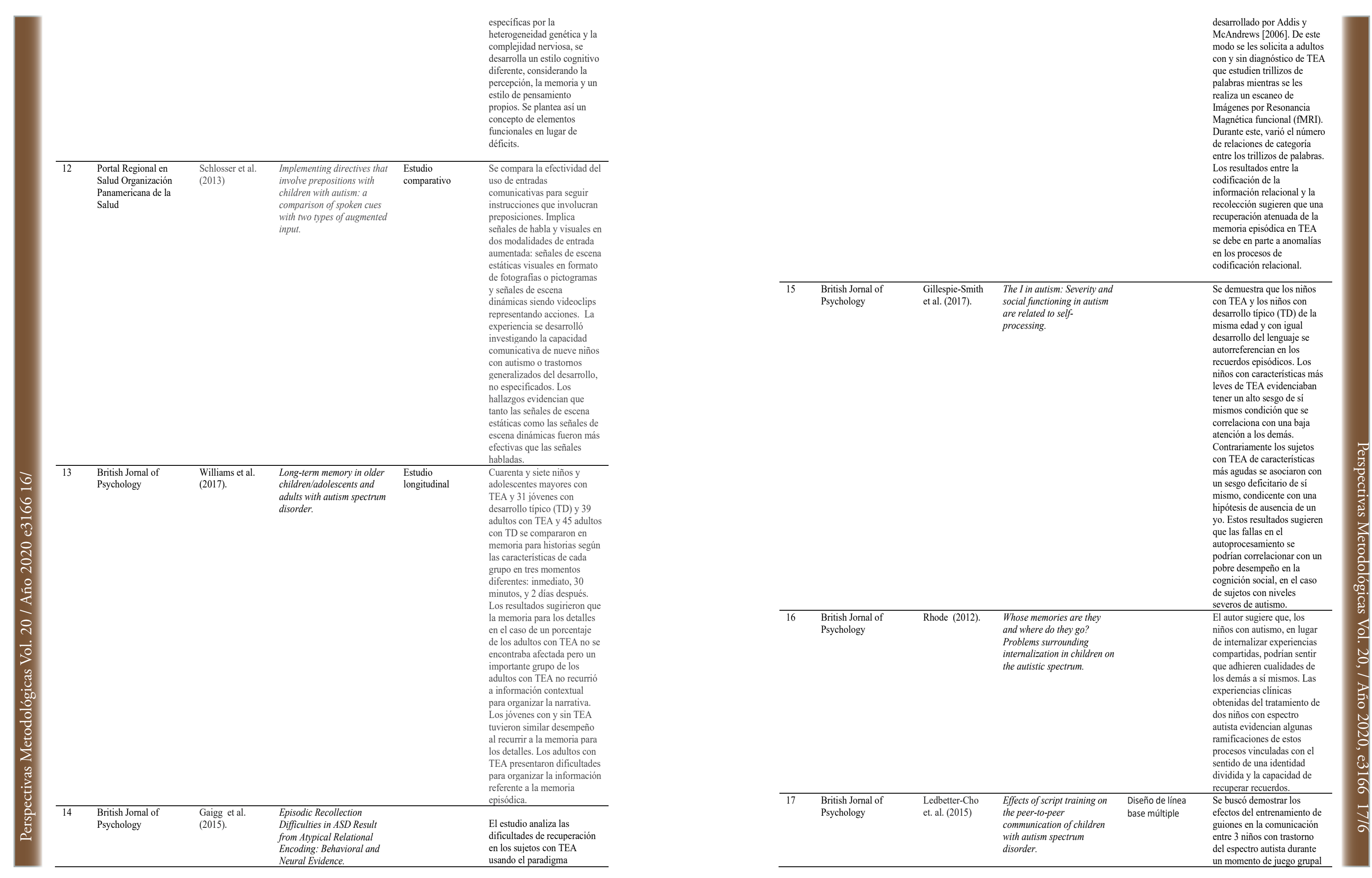

Revista Perspectivas Metodológicas | Universidad Nacional de Lanús | ISSN 2618-4125|

Revista Perspectivas Metodológicas | Universidad Nacional de Lanús | ISSN 2618-4125| 
Según lo relevado en los artículos seleccionados distintos autores manifiestan que las narrativas autobiográficas en las personas con TEA suelen orientarse hacia detalles perceptuales con menos probabilidad emocional en sus recuerdos remotos, sin embargo con importantes probabilidades dela implicancia emociona en los recuerdos más cercanos (Brezis, 2015). Esta autora sostiene además que se favorecen las narraciones preestructuradas sobre las vivencias y experiencias emocionales. De ahí que las deficiencias en memoria autobiográfica se corresponden con dificultades subyacentes en la integración y comprensión emocional (Brezis, 2015). Este estudio se refuerza con el desarrollado por Williams (2017) $\mathrm{El}$ investigador comparó la memoria en la narrativa de historias a un $\mathrm{n}=162$. Entre ellos se encontraban 47 nińos y adolescentes con TEA y 31 jóvenes con desarrollo típico (TD), además de 39 adultos con TEA y 45 adultos con TD. Las historias, adecuadas para cada grupo, se analizaron en tres intervalos de tiempo, de forma inmediata, pasado 30 minutos y después de 2 días. En los resultados se observaron que la memoria para los detalles en el caso de la mayoría de los adultos con TEA no se percibió afectada. Sin embargo, este mismo grupo mostró deficiencias ante el uso de estrategias organizadoras de la memoria episódica. Los jóvenes con TEA tuvieron serias dificultades para el recuerdo pasados los dos días a compararse con el grupo de desarrollo típico. En el caso de los adultos con TEA manifestaron pobreza de desempeńo en el recuerdo de detalles y también para el recuerdo de información relevante contextual para crear la secuencia de la narrativa. Esto hallazgos dejan en evidencia la deficiencia en los tres intervalos de tiempo para e recuerdo. A partir de estos resultados se sugirió también una significativa disocia- ción entre el autorreferenciamiento y la comunicación social (Williams, 2017).

Haciendo un comparativo con Goddard (2014), esta autora sostiene los mismos hallazgos que los referenciados por Wiliams. A través de un estudio en el que se avaluó la memoria autobiográfica en 63 niños de edades comprendidas entre 8 a 17 años con trastorno del espectro autista se buscó indagar sobre la recuperación de eventos específicos del pasado reciente y remoto y memorias semánticas versus episódicas de diferentes momentos de la vida. El estudio se valió de un grupo control de 63 niños de desarrollo típico en igual edad, sexo, coeficiente intelectual y capacidad verbal. En el caso del grupo con TEA se evidenciaron deficiencias en la recuperación del pasado remoto. Hubo inconvenientes en la recuperación de recuerdos episódicos y semánticos en os diferentes momentos de la vida. Se concluyó también que los niños con autismo tenían pocas probabilidades de hacer referencia a los aspectos emocionales en a recuperación de recuerdos remotos, sin embargo, sí podían describir emociones en sus recuerdos cercanos, así como los resultados de Brezis, mencionados en el párrafo anterior.

El estudio de Almeida (2015) apoya este resultado también. El investigador realizó un estudio similar en 27 nińos de 6 a 15 ańos con trastorno del espectro autista y 32 niños con desarrollo típico. Se realizaron entrevistas sobre determinadas actividades primero a las dos semanas y luego a los 2 meses. Las entrevistas se categorizaron sobre tres aspectos, la fidelidad sobre el evento, la cantidad de detalles narrativos y la precisión de la narrativa del recuerdo. Los resultados evidenciaron que los niños con TEA ofrecen testimonio significativo y confiable sobre una determinada 
experiencia, pero varios detalles de los reportes se desvanecen con el transcurso del tiempo. Sin embargo, no se evidenciaron diferencias significativas entre los niños con TEA como los niños con desarrollo típico (Almeida, 2015). Un resultado similar consiguió Anger (2019). En este caso, el estudio se llevó a cabo con 16 nińos con TEA y 16 niños con desarrollo típico de entre 10 y 18 años y se implementó considerando un pasado remoto, un pasado reciente, un futuro reciente y un futuro remoto. Las diferencias observadas se relacionan con las diferencias manifestadas entre los grupos sobre detalles sensoriales acerca grupos sobre detalles sensoriales acerca
de recuerdos del pasado remoto, siendo menores en el grupo con TEA. Sucedió la misma situación al reportar elementos separadamente tales como el color, olores, sonidos, intensidad y sensación táctil. Sin embargo, esta investigación se valió de análogos visuales para ayudar a los niños en la reconstrucción del recuerdo, resultando en un desempeño similar al grupo control. Los resultados evidenciaron un beneficio significativo con el uso de análogos visuales para apoyar la recuperación de episodios autobiográficos, hipótesis ya defendida por Bowler et al. (1997), quien sostiene que el rendimiento mejora considerablemente al proporcionar apoyos para la recuperación.

Zamoscik también ha estudiado la memoria autobiográfica en personas con autismo. El autor ha encontrado algunas contradicciones en la literatura académica. Por un lado manifiesta que varios relatos autobiográficos proporcionados por sujetos con autismo revelan recuerdos muy fidedignos de eventos en la primera infancia, sin embargo, sostiene, un gran número de estudios experimentales evidenciaron déficits en la memoria de recuerdos autobiográficos.
Con objetivo de analizar esta situación contradictoria, se implementó un cuestionario virtual para indagar acerca de los eventos durante la primera infancia. Se trabajó con dos grupos, un prime grupo estaba compuesto por sujetos con autismo y el segundo grupo estaba conformado por sujetos de desarrollo típico. Para este estudio se utilizó una encuesta en línea realizada con el software testMaker. El enlace se distribuyó a través de cuatro foros vinculados al autismo. En el caso del grupo control, el enlace se compartió en foros y plataformas. Un aspecto destacado del estudio fue ofrecer un sorteo por cuatro vales de libros de 10 euros para promover la participación de los sujetos. El estudio indagó sobre los primeros recuerdos autobiográficos y el primer evento semántico conocido a partir del relato de otro. Los datos recopartir del relato de otro. Los datos reco-
lectados mostraron que las personas con autismo recuerdan eventos sucedidos a una edad más temprana en su infancia y también proporcionaron mayores detalles sensoriales. Según este autor, la evidencia contradice el supuesto de un déficit en la memoria episódica personal en sujetos con autismo. Otro aspecto destacado del estudio es la relevancia del lenguaje en la conformación de la memoria destacándose el rol fundamental de las características sensoriales en los recuerdos (Zamoscik, 2016).

Gaigg (2015) también ha encontrado algunas cuestiones inconclusas en el estudio de la memoria, especialmente sobre las dificultades en la codificación para la recuperación en personas con TEA. Para indagar sobre este aspecto se les solicita a adultos con TEA y un grupo control de desarrollo típico que estudien tríos de palabras al mismo tiempo que se les realizaba un escaneo de Imágenes por Resonancia Magnética funcional (fMRI). Los tríos de palabras se combinaban en diferentes categorías y relaciones. Se evidenció un recuerdo atenuado cuando la recuperación implicaba familiaridad. Pero cuando la recuperación implicaba la recolección en función de las relaciones categoriales en los tríos de palabras en ambos grupos se incrementa el recuerdo. De este modo se sugiere un vínculo estrecho entre las dificultades en la recuperación en personas con espectro autista debido a deficiencias en la codificación para establecer relaciones en la información. Estas dificultades en la codificación también fueron evaluadas por Sedano (2013) quien llegó a la conclusión que en los niños autistas aunque tengan la capacidad de verbalización, existen alteraciones lingüísticas en la comunicación que le son propias a sujetos dentro del espectro autista. Postula que la heterogeneidad genética y la complejidad nerviosa juega un papel central en el estilo cognitivo de estos sujetos, es decir, poseen una percepción, memoria y pensamiento particulares con lo cual construyen una peculiar forma de comunicación, así, en lugar de definirse como déficits podría definirse como procesos cognitivos funcionales para la población en cuestión.

Kostin (2018) refuerza esta idea explicando que la problemática en verbalizar experiencias e impresiones personales, la recuperación aislada de recuerdos biográficos y agrega las dificultades de la identidad son características destacadas en la configuración de la autoconciencia en adolescentes y adultos con espectro autista.

Dos autores que encontraron resultados similares con relación al autoprocesamiento fueron Gillespie Smith (2017) y Wuyum (2020). El primero utilizó una tarea de propiedad para demostrar que los niños con autismo y también los niños con desarrollo típico en edades y capacidad de lenguaje emparejadas $s$ manifiestan autorreferenciamiento en la memoria. Niños con diagnóstico de autismo más leves eran más propensos a tener un alto concepto de sí mismos y esta situación parece correlacionarse con baja atención hacia los otros. Los niños autistas con diagnósticos más severos manifiestan un sesgo de sí mismos más débil en comparación. Esta condición parece correlacionarse con la hipótesis de un selfausente. El autor considera que en la literatura existen discrepancias a este respecto, igualmente acuerda con que un débil autoprocesamiento está relacionado con deficiencias sociales y cognitivas (Gillespie Smith (2017) Wuyum (2020) se refiere entonces al autoprocesamiento. Su objetivo fue indagar las deficiencias en este ámbito y el rol de la acción para promover el autoprocesamiento en niños con TEA. Identificó que niños que presentaban discapacidad intelectual y niños con desarrollo típico tenían una ventaja de memoria autorreferencial a diferencia de los sujetos con autismo. Observó que niños con autismo evidenciaban autorreferenciamiento importante al realizar u observar una determinada acción para indicar la propiedad de artículos. A partir de este resultado definió el rol fundamental de la acción para mejorar cognitivamente el autoprocesamiento en la población en cuestión.

Evidentemente la acción juega un rol fundamental en la comunicación de niños autistas. Schlosser (2013) utilizó un diseño para analizar la comunicación a través de tres tipos de entradas, habla, señales (imágenes, fotografías) de escenas estáticas y señales de escenas dinámicas (videos). Partió del supuesto que el uso de la modalidad visual es un medio eficaz para complementar 
el lenguaje hablado. El propósito de la investigación fue analizar la capacidad de los niños para seguir instrucciones que contengan preposiciones a través de las tres entradas diferentes. Se trabajó con nueve niños con autismo o con trastornos generalizados del desarrollo no especificado. Los resultados evidenciaron mayor efectividad de las señales visuales, tanto las estáticas como las dinámicas sobre el lenguaje hablado, aunque no se evidenciaron diferencias entre las imágenes visuales estáticas y las dinámicas. Entonces, la acción en imágenes es un recurso fuerte en la efectividad de la comunicación en niños con trastorno de espectro autista, así como lo comprobó también Wuyum en el estudio anteriormente detallado. La investigación de Zinc (2016) igualmente, analizó el uso de pictogramas para facilitar la comunicación para la atención preventiva en tratamiento odontológico entre niños autistas y el profesional. La investigación se realizó con 26 niños con TEA entre 5 y 19 ańos, entre ellos, 13 niños ya habían tenido la experiencia previa de tratamiento odontológico y 13 niños con TEA no habían tenido experiencia previa de tratamiento dental. Para esto se implementaron pictogramas de habitación, suelo, silla, dentista, boca bajo y triple, que se condicen con la rutina del consultorio y responden a Programa Son-Rise. Cada pictograma se utilizó tres veces para que el niño logre aceptar las señales, siendo que en el caso de los niños que no tenían experiencia previa necesitaron mayor cantidad de veces de práctica para aceptar y seguir la rutina. La conclusión a la que arribaron los investigadores es que efectivamente el uso de las imágenes facilitaron la comunicación entre el paciente y el profesional en función de llevar adelante el tratamiento preventivo odontológico.
La investigación de Zinc dialoga positivamente con el estudio de Nunes (2015) quien, a través de un trabajo cuasi experimental registró un incremento en las iniciativas de interacción de un alumno con TEA con su profesora. El estudio implicó evaluar la eficacia del protocolo Picture Exchange Communication System (Pecs) y estrategias adaptadas de Aided Modeling Intervention (AMI) para promover el desarrollo de la comunicación de un niño autista de cinco años. La experiencia se registra a partir de una intervención psicopedagógica. La maestra del niño fue el agente mediador en la interacción con el niño preparándola para el uso de los pictogramas de tal modo que vaya incrementando el uso de la estrategia durante el avance de las actividades pedagógicas. Como resultado el alumno mejoró las posibilidades de interacción en el vínculo con su profesora por medio de la implementación de pictogramas.

Swain (2015) también se centró en el análisis de la comunicación en esta población. El autor buscó indagar las habilidades comunicativas sociales para su identificación temprana en niños de 12 meses. Realizó un estudio comparativo en niños con trastorno de espectro autista y niños con desarrollo típico. Su objetivo consistía en identificar si las diferencias en las habilidades comunicativas persistían con el avance de la edad inclusive en niños diagnosticados posteriormente con TEA. Efectivamente os niños con TEA manifiestan habilidades más precarias que los nińos con desarrollo típico, tanto en puntaje referente al aspecto social como al simbólico. Se detectaron también diferencias entre el grupo diagnosticados con TEA tempranamente y el grupo con TEA diagnosticado en edad más avanzad con relación al componente social.
A lo largo del análisis de los diferentes estudios se pudo identificar el rol destacado y fundamental del uso de la comunicación alternativa tanto sea para facilitar y mejorar los mismos procesos comunicativos como para organizar la memoria autobiográfica y funcionar a modo de apoyo para la recuperación.

Autores como Brezis (2015), Williams (2017), Almeida (2015) y Anger (2019) sostienen que las narrativas autobiográficas en las personas con TEA suelen orientarse hacia detalles perceptuales con menos probabilidad emocional en sus recuerdos remotos, sin embargo con importantes probabilidades de la implicancia emocional en los recuerdos más cercanos (Brezis, 2015). Sin embargo también se evidenciaron deficiencias ante el uso de estrategias organizadoras de la memoria episódica, por este motivo, Anger estudió la intervención con análogos visuales para ayudar en la recuperación y ordenamiento de los recuerdos. (Zamoscik, 2016) también relevó la importancia del lenguaje para el recuerdo autobiográfico en la población pertinente.

También se analizó la promoción del autorreferenciamiento durante lo procesos de recuperación en la memoria de eventos. Autores como Kostin (2018) Gillespie-Smith (2017) y Wuyum (2020) acuerdan en la debilidad del autorreferenciamiento durante los recuerdos autobiográficos, sin embargo las ayudas visuales cumplen un rol fundamental para el recupero.

Retomando el artículo de Santamaría y Montoya (2008) que se podría decir, promovió el despertar para esta investigación de revisión, estos autores concluyen sosteniendo que las narrativas autobiográficas se definen como un recurso insustituible para construir la identidad en un momento histórico y cultural determinados, considerando a lenguaje como mediador para su construcción. Sin embargo, en función de lo estudios relevados, en los niños con TEA es necesario valerse de ayudas visuales, esenciales para promover y organizar los recuerdos autobiográficos y reforzar el autoprocesamiento.

Igualmente se evidencia la necesidad de investigaciones específicas sobre el rol mediador de los Sistemas Alternativos y Aumentativos de Comunicación en la recuperación de recuerdos autobiográficos y además la capacidad de autorreferenciamiento en estos recuerdos para promover la interacción social tal como lo mencionaban Nunes (2015) y Zinc (2016).

Se espera ampliar este estudio y realizar nuevas investigaciones vinculadas con las necesidades de un mayor conocimiento sobre el funcionamiento de la memoria autobiográfica y su vínculo con el lenguaje en niños con autismo.

\section{Bibliografía}

- Almeida, T. S, Lamb, M. F.Weis blatt, E. J. (2019). Effects of delay on episodic memory retrieval by children with autism spectrum disorder. Applied Cognitive Psychology, 33(5)

- Asociación Americana de Psiquiatría (2014). Manual diagnóstico y estadistico de los trastornos mentales ( $5 \mathrm{a}$ ed.). Arlington, VA. Asociación Americana de Psiquiatría.

- AsociaciónAmericana de Psiquiatría (2000). Manualdiagnóstico yestadístico de los trastornos mentales. Texto revisado. (4a. ed). Arlington, VA. Asociación Americana de Psiquiatría.

- Asociación Americana de Psiquiatría (1994). Manual diaonóstico yestadístico de los trastornos mentales (4a. ed). Arlington, 
VA. Asociación Americana de Psiquiatría

- Anger M, Wantzen P, Le Vaillant J, Malvy J, Bon L, Guénolé F, Moussaoui E, Barthelemy C, Bonnet-Brilhault F, Eustache F Baleyte J-M and Guillery-Girard B. (2019). Positive Effect of Visual Cuing in Episodic Memory and Episodic Future Thinking in Adolescents With Autism Spectrum Disorder. Front. Psychol, 10(1513)

- Boucher, J. y Bowler, D. (2008). Memory in autism. Theory and evidence. London. Cambrige University Press.

- Bowler, D. M., Matthews, N. J., and Gardiner,J.M. (1997). Asperger's síndrome and memory: similarity to autism but no amnesia. Neuropsychologia 35, 65-70

- Brezis R.S. (2015). Memory integration in the autobiographical narratives of individuals with autism. Front. Hum Neurosci, 9(76).

- Canal Bedia, R. y Rivière, A. (1993). La conducta comunicativa de los nińos autista en situaciones naturales de interacción. Estudios de Psicología. 50, 49-74.

-Conway, M. A., \& Pleydell-Pearce, C. W. (2000). The construction of autobiographical memories in the self-memory system. Psychological Review, 107(2), 261-288.

-EIrwin, J. \& eBrancazio, L. (2014). Seeing to hear? Patterns of gaze to speaking faces in children with autism spectrum disorders. Frontiers in Psychology, 5, 1664-1078.

- Gaigg, S., Bowlwr, D., Ecker, Ch. Calvo-Merino, B., Murphy, D. (2015). Episodic Recollection Difficulties in ASD Result from Atypical Relational Encoding: Behavioral and Neural Evidence. Autism Research. INSAR, 8(3), 317-327.

- Gillespie-Smith, K., Ballantyne, C. Branigan, H. P., Turk, D. J., Cunningham, SH. J. (2017). The I in autism: Severity and social functioning in autism are re- lated to self-processing. British Journal of Developmental Psychology, 36(1)

- Goddard, L., Dritschel, B., Robinson, S., and Howlin, P. (2014). Development of autobiographical memory in children with autism spectrum disorders: deficits, gains, and predictors of performance. Dev Psychopathol. 26, 215-228.

- Godoy Echiburú, G., Manghi Haquin, D., Soto Cardenas, G., \& Aranda Godoy, I. (2015). Recursos Comunicativos de un Joven con Autismo: Enfoque Multimodal para Mejorar la Calidad deVida. Actualidades Investigativas en Educación, 15(1), 249-272.

- Guzmán, G., Putrino, N., Martínez F., Quiroz, N. (2017). Nuevas tecnologías: Puentes de comunicación en el trastorno del espectro autista (TEA). Terapia psicológica, 35(3), 247-258.

- Hare, D. J., Mellor, C., y Azmi, S. (2007). Episodic memory in adults with autistic spectrum disorders: Recall for self- versus other experienced events. Research in Developmental Disabilities, 28, 317-329

- Hudson, J. A. (1990). The emergence of autobiographical memory in mother-child conversation. En R. Fivush y J. Hudson (Eds.), Knowing and remembering in young children. Cambridge: Cambridge University Press. 166-196.

- Kostin I.(2018). Approaches and Techniques for the Development of Self-Awareness in Adolescents and Young People with ASD. Autism and DevelopmentalDisorders 16(4), 12-16.

- Lacroix, A., Famelart, N., Guidetti, M. (2016). Language and emotional abilities in children with Williams syndrome and children with autism spectrum disorder: similarities and differences. Pediatric Health, Medicine and Therapeutics, 1, 89-97.

- Ledbetter-Cho, K., Lang, R., Davenport, K., Moore, M., Lee, A., Howell, A. Drew,
C., Dawson, D., Charlop, M. Falconata T., O`Reilly, M. (2015). Effects of script training on the peer-to-peer communication of children with autism spectrum disorder. Jornal of Applied Behavior Analysis 48(4) 785-799.

- Millward, C., Powell, S., Messer, D., y Jordan, R. (2000). Recall for self and othe in autism: Childrens memory for events experienced by themselves and 381 their peers. Journal of Autism and Developmental Disorders, 30, 15-28.

-Nelson, K. (2003). Narrative and self, myth and memory: Emergence of the cultural self. En R. Fivush \& C. A. Haden (Eds.), Autobiographicalmemory and the construction of a narrative self. Developmental and cultural perspectives (pp. 3-29). Mahwah, N.J.: Lawrence Erlbaum Associates.

- Nelson, K. (1993) Psychological and social origins of autobiographical memory. Psychological Science, 4, 7-14

- Neisser, U. (1988). Five kinds of selfknowledge. Philosophical Psychology, 1 35-59.

- Nunes, D. R. de P., Santos, L. B. dos. (2015). Mesclando práticas em Comunicação Alternativa: caso de uma criança com autismo. Psicologia Escolar e Educacional 19(1), 59-69.

- Powell, S. D., y Jordan, R. R. (1993) Being subjective about autistic thinking and learning to learn. Educational Psychology, 13, 359-370.

- Rhode, M. (2012) Whose memories are they and where do they go? Problems surrounding internalization in children on the autistic spectrum. The International Journal of Psychoanalysis, 93(2), 355-376.

- Reese, E. y Fivush, R. (1993). Parental styles of talking about the past. Developmental Psychology, 29, 596-606.
Santamaría, A. y Montoya, E. M. (2008). La memoria autobiográfica: el encuentro entre la memoria, el yo y el lenguaje. Estudios depsicología.333-350.

- Schlosser, R. W., Laubscher, E., Sorce, J., Koul, R., Flynn, S., Hotz, L., Abramson, J., Fadie, H., Shane, H. (2013). Implementing directives that involve prepositions with children with autism: a comparison of spoken cues with two types of augmented input. Augment Altern Commun, 29(2) $132-45$.

- Sedano, M. B. (2013). Modelo de comunicación autista desde los lineamientos metodológicos de la teoría cognitiva sistémica de la comunicación. Apuntes de Ciencia \& Sociedad, 3(1)

- Seijas Gómez, R. (2015). Atención, memoria y funciones ejecutivas en los trastornos del espectro autista: ¡cuánto hemos avanzado desde Leo Kanner?. Revista de la Asociación Española de Neuropsiquiatría, 35(127), 573-586.

- Solcoff, K. (2011). El origen de la Memoria Episódica y de Control de Fuente: Su relación con las capacidades de Teoría de la Mente (Tesis doctoral). Universidad Autónoma de Madrid, Madrid.

- Swain, N. R., Eadie, P. A., Prior, M R., Reilly, Sh. (2015). Assessing early communication skills at 12 months: retrospective study of Autism Spectrum Disorder. International. Journal of Language \& Communication Disorders, $50(4)$

- Tamarit, J. (1993). ¿Qué son los sistemas alternativos de comunicación?En Sotillo (Comp.) Sistemas Alternativos de comunicación. Trotta.

- Tulving, E. (1983). Elements ofepisodic memory. Oxford: Clarendon Press.

- Tulving, E. (1985). Memory and conciousness. Canadian Psychologist, 26, 1-12 
- Tulving, E. (2002). Episodic memory:

From mind to brain. Annual Review of Psychology, 53, 1-25.

- Tulving, E. (2005). Episodic memory and autonoesis: Uniquely human? En $\mathrm{H}$. S. Terrace \&J. Metcalfe (Eds.), The missing link in cognition: Self-knowing consciousness in man and animals. 2-56.

- Williams, D., Minshew, N., Goldstein, G., Mazefsky, C. (2017). Long-term memory in older children/adolescents and adults with autism spectrum disorder. Autism Research, INSAR, 10(9), 1523-1532.

- Williams, D., Nicholson, T., Grainger, C. (2018). The Self-Reference Effect on Perception: Undiminished in Adults with Autism and No Relation to Autism Traits. Autism Research. INSAR, 11(2), 331-341.

- Wuyun, G., Wang, J., Zhang, L., Wang, K., Yi, L., Wu. Y. (2020). Actions Speak Louder Than Words: The Role of Action in Self-Referential Advantage in Children With Autism. Autism Research, 13(5), 810-820.

- Zamoscik V., Mier D., Schmidt S. N. L., Kirsch P. (2016). Early Memories of Individuals on the Autism Spectrum Assessed Using Online Self-Reports. Frontiers in Psychiatry, 7(79).

-Zink, A. G., Baffi Diniz, M., Botti Rodriguez, M.T., Oliveira Guaré, R. (2016). Use of a Picture Exchange Communication System for preventive procedures in individuals with autism spectrum disorder: pilot study. Special Care in dentistry, 36(5), 254-259. 